\title{
Existence of solutions for quasilinear elliptic systems in divergence form with variable growth
}

\author{
Yongqiang Fu and Miaomiao Yang*
}

"Correspondence: 3d07@163.com Department of Mathematics, Harbin Institute of Technology, Harbin, 150001, China

\begin{abstract}
This paper is concerned with the following Dirichlet problem for a quasilinear elliptic system with variable growth: $-\operatorname{div} \sigma(x, u(x), D u(x))=f$ in $\Omega, u(x)=0$ on $\partial \Omega$, where $\Omega \subset \mathbb{R}^{n}$ is a bounded domain. By means of the Young measure and the theory of variable exponent Sobolev spaces, we obtain the existence of solutions in $W_{0}^{1, p(x)}\left(\Omega, \mathbb{R}^{m}\right)$ for each $f \in\left(W_{0}^{1, p(x)}\left(\Omega, \mathbb{R}^{m}\right)\right)^{*}$.
\end{abstract}

Keywords: variable exponent; quasilinear elliptic system; monotone operator; Young measure

\section{Introduction and main result}

In this paper, we consider the Dirichlet problem for the quasilinear elliptic system

$$
\begin{aligned}
& -\operatorname{div} \sigma(x, u(x), D u(x))=f \quad \text { in } \Omega, \\
& u(x)=0 \quad \text { on } \partial \Omega,
\end{aligned}
$$

where $\Omega \subset \mathbb{R}^{n}(n \geq 2)$ is a bounded domain. Here, $f \in\left(W_{0}^{1, p(x)}\left(\Omega, \mathbb{R}^{m}\right)\right)^{*}, p(x)$ is Lipschitz continuous and $1<p_{-}:=\inf _{x \in \bar{\Omega}} p(x) \leq p(x) \leq p_{+}:=\sup _{x \in \bar{\Omega}} p(x)<\infty$, and $\sigma$ satisfies the conditions (H1)-(H3) below. In the following, let $\mathbb{M}^{m \times n}$ denote the real vector space of $m \times n$ matrices equipped with the inner product $M: N=M_{i j} N_{i j}$ (with the usual summation convention).

(H1) (Continuity) $\sigma: \Omega \times \mathbb{R}^{m} \times \mathbb{M}^{m \times n} \rightarrow \mathbb{M}^{m \times n}$ is a Carathéodory function, i.e., $x \mapsto$ $\sigma(x, u, \xi)$ is measurable for every $(u, \xi) \in \mathbb{R}^{m} \times \mathbb{M}^{m \times n}$ and $(u, \xi) \mapsto \sigma(x, u, \xi)$ is continuous for almost every $x \in \Omega$.

(H2) (Growth and coercivity) There exist $c_{1} \geq 0, c_{2}>0,0<a \in L^{p^{\prime}(x)}(\Omega), b \in L^{1}(\Omega)$ and $\frac{p(x)-1}{p(x)}<q(x)<\frac{n(p(x)-1)}{n-p(x)}$, such that

$$
\begin{aligned}
& |\sigma(x, u, \xi)| \leq a(x)+c_{1}\left(|u|^{q(x)}+|\xi|^{p(x)-1}\right) \\
& \sigma(x, u, \xi): \xi \geq-b(x)+c_{2}|\xi|^{p(x)} .
\end{aligned}
$$

(H3) (Monotonicity) $\sigma$ satisfies one of the following conditions: 
(i) For any $x \in \Omega$ and $u \in \mathbb{R}^{m}, \xi \mapsto \sigma(x, u, \xi)$ is $C^{1}$ and monotone, i.e.

$$
(\sigma(x, u, \xi)-\sigma(x, u, \eta)):(\xi-\eta) \geq 0
$$

for any $x \in \Omega, u \in \mathbb{R}^{m}$ and $\xi, \eta \in \mathbb{M}^{m \times n}$.

(ii) There exists a function $W: \Omega \times \mathbb{R}^{m} \times \mathbb{M}^{m \times n} \rightarrow \mathbb{R}$ such that $\sigma(x, u, \xi)=D_{\xi} W(x, u, \xi)$ and $\xi \rightarrow W(x, u, \xi)$ is convex and $C^{1}$.

(iii) $\sigma$ is strictly monotone, i.e., $\sigma$ is monotone and

$$
(\sigma(x, u, \xi)-\sigma(x, u, \eta)):(\xi-\eta)=0 \quad \text { implies } \quad \xi=\eta \text {. }
$$

Our main result is as follows.

Theorem 1.1 If $\sigma$ satisfies conditions (H1)-(H3), then the Dirichlet problem (1.1) has a weak solution $u \in W_{0}^{1, p(x)}\left(\Omega, \mathbb{R}^{m}\right)$ for every $f \in\left(W_{0}^{1, p(x)}\left(\Omega, \mathbb{R}^{m}\right)\right)^{*}$.

After Kováčik and Rákosník first discussed $L^{p(x)}(\Omega)$ and $W^{m, p(x)}(\Omega)$ spaces in [1], a lot of research has been done concerning these kinds of variable exponent spaces, for example, see [2-6] for the properties of such spaces and [7-9] for the applications of variable exponent spaces on partial differential equations. These problems with variable exponent growth possess very complicated nonlinearities, for instance, the $p(x)$-Laplacian operator is inhomogeneous. In recent years, these problems have received considerable attention and raised many difficult mathematical problems. The theory as regards various mathematical problems with $p(x)$-growth conditions has important applications in nonlinear elastic mechanics, imaging processing, electrorheological fluids, and other physics phenomena [10-15].

Condition (H2) states the variable growth and coercivity condition. For the case that $p(x)$ is a constant function, Norbert Hungerbühler [16] studied the problem above. The classical monotone operator methods developed by [17-20] cannot be applied in functions only satisfying the condition in [16]. Inspired by the works mentioned above, we want to extend the result of [16] to the case that $\sigma$ satisfies variable growth conditions. To our knowledge, problem (1.1) with variable growth conditions has never been studied by others.

The classical result of Leray and Lions and other typical monotone operator methods require strict monotonicity or monotonicity in the variables $(u, \xi)$ (see [17-20] and the references therein). In (H3), it is not required that $\sigma$ is strict monotone or monotone in the variables $(u, \xi)$ as had usually been assumed in previous work. We only require that $\xi \mapsto \sigma(x, u, \xi)$ is monotone. Here is an example: $g: \mathbb{R} \rightarrow \mathbb{R}$,

$$
g(x)= \begin{cases}0, & |t| \leq R \\ 1, & |t|>R\end{cases}
$$

For $h<\frac{R}{2}, g_{h}(t)=S_{h}(g(t))=\frac{1}{h}\left(\int_{a}^{t+\frac{h}{2}} g(s) d s-\int_{a}^{t-\frac{h}{2}} g(s) d s\right)=\frac{1}{h} \int_{t-\frac{h}{2}}^{t+\frac{h}{2}} g(s) d s$. For $h^{\prime}=\frac{h}{2}$, set $\tilde{g}(t)=S_{h^{\prime}}\left(g_{h}(t)\right)$. We can take $\sigma(x, u, \xi)=\tilde{g}(|\xi|)|\xi|^{p(x)-2} \xi$. If $|\xi| \leq R-\frac{3 h}{4}, \sigma(\xi)=0$; if $|\xi| \geq$ $R+\frac{3 h}{4}, \sigma(x, u, \xi)=|\xi|^{p(x)-2} \xi$. (H1) and (H2) are satisfied. $\xi \mapsto \sigma(x, u, \xi)$ is monotone and $C^{1}$, 
but not strictly monotone. If (H3)(ii) is assumed, this problem cannot be treated by conventional methods since the gradients of the approximating solutions do not necessarily converge pointwise where $W$ is not strictly convex (and thus $\sigma$ is not strictly monotone). Technically, this can be achieved by considering the Young measure generated by the sequence of gradients of approximating solutions which is inspired by [21].

This paper is organized as follows: In Section 2, several important properties on variable exponent spaces are presented; in Section 3, we give some conclusions concerned with the Young measure in a variable exponent space; in Section 4, we construct the Galerkin approximation sequence; in Section 5, the proof of Theorem 1.1 is given.

\section{Preliminaries}

In this section, we first recall some facts on variable exponent spaces $L^{p(x)}(\Omega)$ and $W^{k, p(x)}(\Omega)$. See $[1,4-6]$ for details.

Let $\mathbf{P}(\Omega)$ be the set of all Lebesgue measurable functions $p: \Omega \rightarrow[1,+\infty]$, where $\Omega \subset \mathbb{R}^{n}$ $(n \geq 2)$ is a nonempty open subset. Denote

$$
\begin{aligned}
& \rho_{p(x)}(u)=\int_{\Omega \backslash \Omega_{\infty}}|u(x)|^{p(x)} d x+\sup _{\Omega_{\infty}}|u(x)|, \\
& \|u\|_{p(x)}=\inf \left\{t>0: \rho_{p(x)}\left(\frac{u}{t}\right) \leq 1\right\},
\end{aligned}
$$

where $\Omega_{\infty}=\{x \in \Omega: p(x)=\infty\}$. The variable exponent Lebesgue space $L^{p(x)}(\Omega)$ is the class of all functions $u$ such that $\rho_{p(x)}\left(t_{0} u\right)<\infty$ for some $t_{0}>0$. $L^{p(x)}(\Omega)$ is a Banach space endowed with the norm (2.2). Equation (2.1) is called the modular of $u$ in $L^{p(x)}(\Omega)$.

For a given $p(x) \in \mathbf{P}(\Omega)$, we define the conjugate function $p^{\prime}(x)$ as

$$
p^{\prime}(x)= \begin{cases}\infty, & \text { if } x \in \Omega_{1}=\{x \in \Omega: p(x)=1\} \\ 1, & \text { if } x \in \Omega_{\infty} \\ \frac{p(x)}{p(x)-1}, & \text { for other } x \in \Omega\end{cases}
$$

Lemma 2.1 ([6]) Let $p \in \mathbf{P}(\Omega)$, then the inequality

$$
\int_{\Omega}|u(x) \cdot v(x)| d x \leq 2\|u\|_{p(x)}\|v\|_{p^{\prime}(x)}
$$

holds for every $u \in L^{p(x)}(\Omega), v \in L^{p^{\prime}(x)}(\Omega)$.

In the following of this section, for every $p \in \mathbf{P}(\Omega)$, we assume $1 \leq p_{-} \leq p(x) \leq p_{+}<\infty$.

Lemma 2.2 ([5]) For any $u \in L^{p(x)}(\Omega)$, we have:

1. If $\|u\|_{p(x)} \geq 1$, then $\|u\|_{p(x)}^{p_{-}} \leq \rho_{p(x)}(u) \leq\|u\|_{p(x)}^{p_{+}}$.

2. If $\|u\|_{p(x)}<1$, then $\|u\|_{p(x)}^{p_{+}} \leq \rho_{p(x)}(u) \leq\|u\|_{p(x)}^{p_{-}}$.

Lemma 2.3 ([5]) If $p_{-}>1, L^{p(x)}(\Omega)$ is reflexive, and the dual space of $L^{p(x)}(\Omega)$ is $L^{p^{\prime}(x)}(\Omega)$.

Lemma 2.4 ([1]) Let $|\Omega|<\infty$, where $|\Omega|$ denotes the Lebesgue measure of $\Omega, p_{1}(x), p_{2}(x) \in$ $\mathbf{P}(\Omega)$, then the necessary and sufficient condition for $L^{p_{2}(x)}(\Omega) \subset L^{p_{1}(x)}(\Omega)$ is that $p_{1}(x) \leq$ $p_{2}(x)$ for almost every $x \in \Omega$, and in this case the embedding is continuous. 
Next $k$ is a given positive integer. Given a multi-index $\alpha=\left(\alpha_{1}, \ldots, \alpha_{n}\right) \in \mathbb{N}^{n}$, we set $|\alpha|=$ $\alpha_{1}+\cdots+\alpha_{n}$ and $D^{\alpha}=D_{1}^{\alpha_{1}} \ldots D_{n}^{\alpha_{n}}$, where $D_{i}=\partial / \partial x_{i}$ is the generalized derivative operator.

The generalized Sobolev space $W^{k, p(x)}(\Omega)$ is the class of functions $u$ on $\Omega$ such that $D^{\alpha} u \in$ $L^{p(x)}(\Omega)$ for every multi-index $\alpha$ with $|\alpha| \leq k . W^{k, p(x)}(\Omega)$ is a Banach space endowed with the norm

$$
\|u\|_{k, p(x)}=\sum_{|\alpha| \leq k}\left\|D^{\alpha} u\right\|_{p(x)} .
$$

By $W_{0}^{k, p(x)}(\Omega)$ we denote the subspace of $W^{k, p(x)}(\Omega)$ which is the closure of $C_{0}^{\infty}(\Omega)$ with respect to the norm $(2.3)$.

For any $u \in W^{1, p(x)}(\Omega)$, define

$$
\|u\|=\inf \left\{t>0: \int_{\Omega} \frac{|\nabla u|^{p(x)}+|u|^{p(x)}}{t^{p(x)}} d x \leq 1\right\},
$$

then $\|\cdot \mid\|$ is an equivalent norm of $W^{1, p(x)}(\Omega)$. If $\Omega$ is a bounded domain, $\|\nabla u\|_{p(x)}$ is an equivalent norm of $W_{0}^{1, p(x)}(\Omega)$.

Lemma $2.5([1])$ The spaces $W^{k, p(x)}(\Omega)$ and $W_{0}^{k, p(x)}(\Omega)$ are separable. Furthermore they are reflexive if $p_{-}>1$.

We denote the dual space of $W_{0}^{k, p(x)}(\Omega)$ by $W^{-k, p^{\prime}(x)}(\Omega)$, then we have

Lemma 2.6 ([1]) Let $p \in \mathbf{P}(\Omega) \cap L^{\infty}(\Omega)$. Then for every $G \in W^{-k, p^{\prime}(x)}(\Omega)$, there exists $g_{\alpha} \in$ $\left\{g_{\alpha} \in L^{p^{\prime}(x)}(\Omega):|\alpha| \leq k\right\}$ such that

$$
G(u)=\sum_{|\alpha| \leq k} \int_{\Omega} D^{\alpha} u(x) g_{\alpha}(x) d x, \quad \forall u \in W_{0}^{k, p(x)}(\Omega) .
$$

The norm of $W^{-k, p^{\prime}(x)}(\Omega)$ is defined as

$$
\|G\|_{-k, p^{\prime}(x)}=\sup \left\{\frac{|G(u)|}{\|u\|_{k, p(x)}}: u \in W^{k, p(x)}(\Omega) \backslash\{0\}\right\} .
$$

Lemma 2.7 ([4]) Let $\Omega$ be a domain in $\mathbb{R}^{n}$ with cone property. If $p: \bar{\Omega} \rightarrow \mathbb{R}$ is Lipschitzcontinuous and $1<p_{-} \leq p^{+}<\frac{n}{k}, q: \bar{\Omega} \rightarrow \mathbb{R}$ is measurable and satisfies $p(x) \leq q(x) \leq p^{*}(x):=$ $\frac{n p(x)}{n-k p(x)}$ for almost every $x \in \bar{\Omega}$, then there is a continuous embedding $W^{k, p(x)}(\Omega) \hookrightarrow L^{q(x)}(\Omega)$.

Lemma 2.8 ([4]) Let $\Omega$ be a domain in $\mathbb{R}^{n}$ with cone property. If $p: \bar{\Omega} \rightarrow \mathbb{R}$ is continuous and $1<p_{-} \leq p^{+}<\frac{n}{k}$, then for any measurable function $q(x)$ defined in $\Omega$ with $p(x) \leq q(x)$ for almost every $x \in \bar{\Omega}$, and $\inf _{x \in \bar{\Omega}}\left(p^{*}(x)-q(x)\right)>0$, there is a continuous compact embedding $W^{k, p(x)}(\Omega) \hookrightarrow L^{q(x)}(\Omega)$.

Lemma 2.9 ([1]) Let $h: \Omega \times \mathbb{R}^{m} \rightarrow \mathbb{R}, m \in \mathbb{N}$, satisfy the Carathéodory conditions, $p_{i}, r \in$ $\mathbf{P}(\Omega) \cap L^{\infty}(\Omega), i=1, \ldots, m$. If there exist a nonnegative function $g \in L^{r(x)}(\Omega)$ and a constant $c>0$ such that

$$
|h(x, \xi)| \leq g(x)+c \sum_{i=1}^{m}\left|\xi_{i}\right|^{\frac{p_{i}(x)}{r(x)}}
$$


for every $\xi \in \mathbb{R}^{m}$ and almost every $x \in \Omega$, then the Nemyckii operator $H: L^{p_{1}(x)}(\Omega) \times \cdots \times$ $L^{p_{m}(x)}(\Omega) \rightarrow L^{r(x)}(\Omega)$, defined by

$$
H\left(u_{1}, \ldots, u_{m}\right)(x)=h\left(x, u_{1}(x), \ldots, u_{m}(x)\right), \quad x \in \Omega
$$

is continuous and bounded.

\section{The Young measure generated by sequences in variable exponent space}

Weak convergence is a basic tool of modern nonlinear analysis, because it has the same compactness properties as the convergence in finite dimensional spaces (see [22]). But this notion does not behave as we desire with respect to nonlinear functionals and operators. The Young measure is a device to overcome these difficulties. For the details we refer to [21-24]. Inspired by these works, we will show our conclusions on Young measures in variable exponent space. In what follows, we denote $f^{-}(x)=\max \{-f(x), 0\}$ as the negative part of $f(x)$. First, we recall the definition of Young measures and some lemmas.

Definition 3.1 ([22]) Assume that the sequence $\left\{f_{k}\right\}_{k=1}^{\infty}$ is bounded in $L^{\infty}\left(\Omega ; \mathbb{R}^{m}\right)$. Then there exist a subsequence $\left\{f_{k_{j}}\right\}_{j=1}^{\infty} \subset\left\{f_{k}\right\}_{k=1}^{\infty}$ and a Borel probability measure $v_{x}$ on $\mathbb{R}^{m}$ for a.e. $x \in \Omega$, such that for each $F \in C\left(\mathbb{R}^{m}\right)$ we have

$$
F\left(f_{k_{j}}\right) \stackrel{*}{\rightarrow} \bar{F} \quad \text { weakly* in } L^{\infty}(\Omega),
$$

where

$$
\bar{F}(x) \equiv \int_{\mathbb{R}^{m}} F(y) d v_{x}(y) \quad(\text { a.e. } x \in \Omega)
$$

We call $\left\{v_{x}\right\}_{x \in U}$ the family of Young measure associated with the subsequence $\left\{f_{k_{j}}\right\}_{j=1}^{\infty}$.

Lemma 3.2 ([21]) Let $\Omega \subset \mathbb{R}^{n}$ be Lebesgue measurable (not necessarily bounded) and $z_{j}$ : $\Omega \rightarrow \mathbb{R}^{m}, j=1,2, \ldots$, be a sequence of Lebesgue measurable functions. Then there exist a subsequence $z_{k}$ and a family $\left\{v_{x}\right\}_{x \in \Omega}$ of nonnegative Radon measures on $\mathbb{R}^{n}$, such that

(i) $\left\|v_{x}\right\|:=\int d v_{x} \leq 1$ for almost every $x \in \Omega$.

(ii) $\varphi\left(z_{k}\right) \stackrel{*}{\rightarrow} \bar{\varphi}$ weakly* in $L^{\infty}(\Omega)$ for any $\varphi \in C_{0}\left(\mathbb{R}^{m}\right)$, where $\bar{\varphi}(x)=\left\langle v_{x}, \varphi\right\rangle$ and $C_{0}\left(\mathbb{R}^{m}\right)=\left\{\varphi \in C\left(\mathbb{R}^{m}\right): \lim _{|z| \rightarrow \infty}|\varphi(z)|=0\right\}$.

(iii) If for any $R>0$

$$
\lim _{L \rightarrow \infty} \sup _{k \in \mathbb{N}} \operatorname{meas}\left\{x \in \Omega \cap B(0, R):\left|z_{k}(x)\right| \geq L\right\}=0,
$$

then $\left\|v_{x}\right\|=1$ for almost every $x \in \Omega$, and for any measurable $A \subset \Omega$ we have $\varphi\left(z_{k}\right) \rightarrow \bar{\varphi}=$ $\left\langle v_{x}, \varphi\right\rangle$ weakly in $L^{1}(A)$ for continuous $\varphi$ provided the sequence $\varphi\left(z_{k}\right)$ is weakly precompact in $L^{1}(A)$.

Lemma 3.2 is the fundamental theorem of the Young measure. A family $\left\{v_{x}\right\}_{x \in \Omega}$ satisfying (i)-(ii) always exists and $v_{x}$ is a probability measure if equation (3.1) holds. Lemma 3.2 has useful applications in nonlinear PDE theory. The following lemmas are useful for us. 
Lemma 3.3 ([24]) If $|\Omega|<\infty$ and $v_{x}$ is the Young measure generated by the sequence $\left\{u_{j}\right\}$, then we have $u_{j} \rightarrow u$ in measure if and only if $v_{x}=\delta_{u(x)}$ for almost every $x \in \Omega$.

Lemma 3.4 ([24]) Let $|\Omega|<\infty$. If the sequences $\left\{u_{j}\right\}$ and $\left\{v_{j}\right\}$ generate the Young measures $\delta_{u(x)}$ and $v_{x}$, respectively, where $u_{j}: \Omega \rightarrow \mathbb{R}^{m}$ and $v_{j}: \Omega \rightarrow \mathbb{R}^{d}$, then $\left\{\left(u_{j}, v_{j}\right)\right\}$ generates the Young measure $\delta_{u(x)} \otimes v_{x}$.

Lemma 3.5 ([21]) Let $F: \Omega \times \mathbb{R}^{m} \times \mathbb{M}^{m \times n} \rightarrow \mathbb{R}$ be a Carathéodory function and $\left\{u_{k}\right\}$ be a sequence of measurable functions, where $u_{k}: \Omega \rightarrow \mathbb{R}^{m}$, such that $u_{k} \rightarrow u$ in measure and $D u_{k}$ generates the Young measure $v_{x}$. Then

$$
\liminf _{k \rightarrow \infty} \int_{\Omega} F\left(x, u_{k}(x), D u_{k}(x)\right) d x \geq \int_{\Omega} \int_{\mathbb{M}^{m \times n}} F(x, u, \lambda) d v_{x}(\lambda) d x,
$$

provided that the negative part $F^{-}\left(x, u_{k}(x), D u_{k}(x)\right)$ is equiintegrable.

Theorem 3.6 If the sequence $\left\{u_{j}\right\}$ is bounded in $L^{p(x)}\left(\Omega, \mathbb{R}^{m}\right)$, then there is a Young measure $v_{x}$ generated by $\left\{u_{j}\right\}$ satisfying $\left\|v_{x}\right\|=1$ and the weak $L^{1}$-limit of $\left\{u_{j}\right\}$ is $\int_{\mathbb{R}^{m}} \lambda d v_{x}(\lambda)$.

Proof It suffices to prove that $\left\{u_{j}\right\}$ satisfies equation (3.1) in Lemma 3.2. By Lemma 2.2, there is $C \geq 0$, for any $R>0$,

$$
\begin{aligned}
C & \geq \int_{\Omega}\left|u_{j}\right|^{p(x)} d x \\
& \geq \int_{\left\{x \in \Omega \cap B(0, R):\left|u_{j}(x)\right| \geq L\right\}}\left|u_{j}\right|^{p(x)} d x \\
& \geq L^{p_{-}} \operatorname{meas}\left\{x \in \Omega \cap B(0, R):\left|u_{j}(x)\right| \geq L\right\} .
\end{aligned}
$$

Thus

$$
\sup _{j \in \mathbb{N}} \operatorname{meas}\left\{x \in \Omega \cap B(0, R):\left|u_{j}(x)\right| \geq L\right\} \leq \frac{C}{L^{p_{-}}} \rightarrow 0, \quad \text { as } L \rightarrow \infty .
$$

According to Lemma 3.2(iii), $\left\|v_{x}\right\|=1$. By Lemma 2.3, $L^{p(x)}\left(\Omega, \mathbb{R}^{m}\right)$ is reflexive, then there is a subsequence of $\left\{u_{j}\right\}$ (still denoted by $\left\{u_{j}\right\}$ ) weakly convergent in $L^{p(x)}\left(\Omega, \mathbb{R}^{m}\right)$. Moreover $\left\{u_{j}\right\}$ weakly converges in $L^{1}\left(\Omega, \mathbb{R}^{m}\right)$. By Lemma 3.2(iii), taking $\varphi$ as the identity mapping $I$, we have $u_{j} \rightarrow\left\langle v_{x}, I\right\rangle=\int_{\mathbb{R}^{m}} \lambda d v_{x}(\lambda)$ weakly in $L^{1}\left(\Omega, \mathbb{R}^{m}\right)$.

Theorem 3.7 Let $|\Omega|<\infty$. If $u_{k} \rightarrow u$ in $W_{0}^{1, p(x)}\left(\Omega, \mathbb{R}^{m}\right)$, then the sequence $\left\{\left(u_{k}, D u_{k}\right)\right\}$ generates the Young measure $\delta_{u(x)} \otimes v_{x}$. Moreover, for almost every $x \in \Omega, v_{x}$ is a probability measure and satisfies $\left\langle v_{x}, I\right\rangle=D u(x)$.

Proof Since $u_{k} \rightarrow u$ in $W_{0}^{1, p(x)}\left(\Omega, \mathbb{R}^{m}\right),\left\{u_{k}\right\}$ is bounded in $W_{0}^{1, p(x)}\left(\Omega, \mathbb{R}^{m}\right)$. By Lemma 2.8 ,

$$
u_{k} \rightarrow u \quad \text { in } L^{p(x)}\left(\Omega, \mathbb{R}^{m}\right) \quad \text { and } \quad u_{k} \rightarrow u \quad \text { in measure. }
$$

According to Theorem 3.6 and Lemma 3.3, $\left\{u_{k}\right\}$ generates the Young measure $\delta_{u(x)}$ and $\left\{D u_{k}\right\}$ generates the Young measure $v_{x}$ such that $v_{x}$ is a probability measure. By Lemma 3.4, the sequence $\left\{\left(u_{k}, D u_{k}\right)\right\}$ generates the Young measure $\delta_{u(x)} \otimes v_{x}$. 
Since $u_{k} \rightarrow u$ in $W_{0}^{1, p(x)}\left(\Omega, \mathbb{R}^{m}\right)$ and $u_{k} \rightarrow u$ in $L^{p(x)}\left(\Omega, \mathbb{R}^{m}\right)$, we have

$$
D u_{k} \rightarrow D u \quad \text { in } L^{p(x)}\left(\Omega, \mathbb{M}^{m \times n}\right)
$$

moreover

$$
D u_{k} \rightarrow D u \quad \text { in } L^{1}\left(\Omega, \mathbb{M}^{m \times n}\right) .
$$

By Theorem 3.6, we can infer that $\left\langle v_{x}, I\right\rangle=D u(x)$.

\section{Galerkin approximation}

Let $V_{1} \subset V_{2} \subset \cdots \subset W_{0}^{1, p(x)}\left(\Omega, \mathbb{R}^{m}\right)$ be a sequence of finite dimensional subspaces with the property that $\bigcup_{i \in \mathbb{N}} V_{i}$ is dense in $W_{0}^{1, p(x)}\left(\Omega, \mathbb{R}^{m}\right)$. We define the operator

$$
\begin{aligned}
& J: W_{0}^{1, p(x)}\left(\Omega, \mathbb{R}^{m}\right) \rightarrow\left(W_{0}^{1, p(x)}\left(\Omega, \mathbb{R}^{m}\right)\right)^{*}, \\
& u \mapsto\left(w \mapsto \int_{\Omega} \sigma(x, u(x), D u(x)): D w d x-\langle f, w\rangle\right),
\end{aligned}
$$

where $\langle$,$\rangle denotes the dual pairing of \left(W_{0}^{1, p(x)}\left(\Omega, \mathbb{R}^{m}\right)\right)^{*}$ and $W_{0}^{1, p(x)}\left(\Omega, \mathbb{R}^{m}\right)$, and $\sigma$ satisfies (H1)-(H3).

Lemma 4.1 For every $u \in W_{0}^{1, p(x)}\left(\Omega, \mathbb{R}^{m}\right)$, the functional $J(u)$ is linear and bounded.

Proof It is easy to see that $J(u)$ is linear. By the growth condition in (H2) and Lemma 2.7,

$$
\int_{\Omega}|\sigma(x, u, D u)|^{p^{\prime}(x)} d x \leq C \int_{\Omega}\left(|a(x)|^{p^{\prime}(x)}+|u|^{q(x) p^{\prime}(x)}+|D u|^{(p(x)-1) p^{\prime}(x)}\right) d x<\infty .
$$

By Lemma 2.1 and Lemma 2.2, for each $w \in W_{0}^{1, p(x)}\left(\Omega, \mathbb{R}^{m}\right)$

$$
\begin{aligned}
|\langle J(u), w\rangle| & =\left|\int_{\Omega} \sigma(x, u, d u): D w d x-\langle f, w\rangle\right| \\
& \leq \int_{\Omega}|\sigma(x, u, d u)| \cdot|D w| d x+|\langle f, w\rangle| \\
& \leq 2\||\sigma(x, u, D u)|\|_{p^{\prime}(x)}\||D w|\|_{p(x)}+\|f\|_{-1, p^{\prime}(x)} \cdot\|w\| \mid \\
& \leq C\|\mid w\| .
\end{aligned}
$$

This implies that $J(u)$ is bounded.

Lemma 4.2 The restriction of J to a finite linear subspace of $W_{0}^{1, p(x)}\left(\Omega, \mathbb{R}^{m}\right)$ is continuous.

Proof By the continuity assumption (H1) and the growth condition in (H2),

$$
\begin{aligned}
\left\|J\left(u_{k}\right)-J(u)\right\| & =\sup _{\|w\|=1}\left|\left\langle J\left(u_{k}\right), w\right\rangle-\langle J(u), w\rangle\right| \\
& =\sup _{\|w\|=1}\left|\int_{\Omega} \sigma\left(x, u_{k}, D u_{k}\right): D w d x-\int_{\Omega} \sigma(x, u, D u): D w d x\right| \\
& \leq C\left\|\left|\sigma\left(x, u_{k}, D u_{k}\right)-\sigma(x, u, D u)\right|\right\|_{p^{\prime}(x)} .
\end{aligned}
$$


Observe that

$$
|\sigma(x, u, D u)| \leq a(x)+C_{1}\left(|u|^{\frac{q(x) p^{\prime}(x)}{p^{\prime}(x)}}+|D u|^{\frac{(p(x)-1) p^{\prime}(x)}{p^{\prime}(x)}}\right) .
$$

Thus by Lemma 2.7 and Lemma 2.9 , we can get the conclusion.

Let us fix some $k$ and assume that the dimension of $V_{k}$ is $r$ and $\phi_{1}, \ldots, \phi_{r}$ is a basis of $V_{k}$. For simplicity, we write $\sum_{i=1}^{r} a^{i} \phi_{i}=a^{i} \phi_{i}$. Then we define

$$
G: \mathbb{R}^{r} \rightarrow \mathbb{R}^{r}, \quad\left(\begin{array}{c}
a^{1} \\
a^{2} \\
\vdots \\
a^{r}
\end{array}\right) \mapsto\left(\begin{array}{c}
\left\langle J\left(a^{i} \phi_{i}\right), \phi_{1}\right\rangle \\
\left\langle\left(a^{i} \phi_{i}\right), \phi_{2}\right\rangle \\
\vdots \\
\left\langle J\left(a^{i} \phi_{i}\right), \phi_{r}\right\rangle
\end{array}\right)
$$

Lemma 4.3 $G$ is continuous and

$$
G(a) \cdot a \rightarrow \infty \quad \text { as }\|a\|_{\mathbb{R}^{r}} \rightarrow \infty
$$

where $\cdot$ denotes the inner product of two vectors in $\mathbb{R}^{r}$.

Proof In order to prove that $G$ is continuous, it is sufficient to show that $G\left(a_{l}\right) \rightarrow G\left(a_{0}\right)$ in $\mathbb{R}^{r}$ as $a_{l} \rightarrow a_{0}$ in $\mathbb{R}^{r}$. Let $u_{l}=a_{l}^{i} \phi_{i} \in V_{k}, u_{0}=a_{0}^{i} \phi_{i} \in V_{k}$. Then $\left\|a_{l}\right\|_{\mathbb{R}^{r}}$ is equivalent to $\left\|u_{l}\right\| \|$ and $\left\|a_{0}\right\|_{\mathbb{R}^{r}}$ is equivalent to $\left\|u_{0}\right\|$. We have

$$
\begin{aligned}
\left|\left(G\left(a_{l}\right)-G(a)\right)_{j}\right| & =\left|\left\langle J\left(a_{l}^{i} \phi_{i}\right)-J\left(a_{0}^{i} \phi_{i}\right), \phi_{j}\right\rangle\right| \\
& \leq\left\|J\left(u_{l}\right)-J\left(u_{0}\right)\right\|_{-1, p^{\prime}(x)} \cdot\left\|\phi_{j}\right\| .
\end{aligned}
$$

Since $J$ is continuous on finite dimensional subspaces, we can get the continuity of $G$.

Moreover taking $u=a^{i} \phi_{i} \in V_{k},\|a\|_{\mathbb{R}^{r}} \rightarrow \infty$ is equivalent to $\|u\| \rightarrow \infty$, thus

$$
\begin{aligned}
G(a) \cdot a & =\left\langle J\left(a^{i} \phi_{i}\right), a^{i} \phi_{i}\right\rangle \\
& =\langle J(u), u\rangle \\
& =\int_{\Omega} \sigma(x, u, D u): D u d x-\langle f, u\rangle \\
& \geq \int_{\Omega}-b(x)+c_{2}|D u|^{p(x)} d x-\|f\|_{-1, p^{\prime}(x)} \cdot\|u\| \| \\
& \geq C+C\|u\|^{p_{-}}-\|f\|_{-1, p^{\prime}(x)} \cdot\|u\| \rightarrow \rightarrow \infty,
\end{aligned}
$$

as $\|u\| \rightarrow \infty$.

Lemma 4.4 For any $k \in \mathbb{N}$, there exists $u_{k} \in V_{k}$ such that

$$
\left\langle J\left(u_{k}\right), v\right\rangle=0, \quad v \in V_{k} .
$$


Proof By Lemma 4.3, there exists $R>0$ such that for any $a \in \partial B_{R}(0) \subset \mathbb{R}^{r}$ we have $G(a) \cdot a>0$ and the topological argument [25] shows that $G(x)=0$ has a solution $x \in B_{R}(0)$. Hence, for each $k$ there exists $u_{k} \in V_{k}$ such that our conclusion holds.

\section{Proof of Theorem 1.1}

In this section, first we give some lemmas for $\sigma$ satisfying $(\mathrm{H} 1)-(\mathrm{H} 3)$. Then we prove Theorem 1.1.

Lemma 5.1 If $u_{k} \rightarrow u$ in $W_{0}^{1, p(x)}\left(\Omega, \mathbb{R}^{m}\right)$, $\sigma$ satisfies $(\mathrm{H} 1)-(\mathrm{H} 3)$ and $\left\{D u_{k}\right\}$ generates the Young measure $v_{x}$, then the following inequality holds:

$$
\int_{\Omega} \int_{\mathbb{M}^{m \times n}} \sigma(x, u, \lambda): \lambda d v_{x}(\lambda) d x \leq \int_{\Omega} \int_{\mathbb{M}^{m \times n}} \sigma(x, u, \lambda): D u d v_{x}(\lambda) d x .
$$

Furthermore $\sigma\left(x, u_{k}(x), D u_{k}(x)\right)$ is equiintegrable.

Proof Let us consider the sequence

$$
I_{k}:=\left(\sigma\left(x, u_{k}, D u_{k}\right)-\sigma(x, u, D u)\right):\left(D u_{k}-D u\right) .
$$

We will use Lemma 3.5 giving

$$
X:=\liminf _{k \rightarrow \infty} \int_{\Omega} I_{k} d x \geq \int_{\Omega} \int_{\mathbb{M}^{m \times n}} \sigma(x, u, \lambda):(\lambda-D u) d v_{x}(\lambda) d x
$$

So we have to establish the equiintegrability of negative part $I_{k}^{-}$of $I_{k}$. We write $I_{k}$ in the form

$$
\begin{aligned}
I_{k}= & \sigma\left(x, u_{k}, D u_{k}\right): D u_{k}-\sigma\left(x, u_{k}, D u_{k}\right): D u \\
& -\sigma(x, u, D u): D u_{k}+\sigma(x, u, D u): D u=: I_{k, 1}+I_{k, 2}+I_{k, 3}+I_{k, 4} .
\end{aligned}
$$

To get the equiintegrability of the sequence $\left\{I_{k, 2}\right\}$, we take a measurable subset $\Omega^{\prime} \subset \Omega$ and by Lemma 2.1

$$
\begin{aligned}
\int_{\Omega^{\prime}}\left|\sigma\left(x, u_{k}, D u_{k}\right): D u\right| d x & \leq \int_{\Omega^{\prime}}\left|\sigma\left(x, u_{k}, D u_{k}\right)\right| \cdot|D u| d x \\
& \leq 2\left\|\left|\sigma\left(x, u_{k}, D u_{k}\right)\right|\right\|_{p^{\prime}(x), \Omega^{\prime}} \cdot\||D u|\|_{p(x), \Omega^{\prime}}
\end{aligned}
$$

Since $\left\{u_{k}\right\}$ is bounded in $W_{0}^{1, p(x)}\left(\Omega, \mathbb{R}^{m}\right)$, by the growth condition in (H2) and Lemma 2.7,

$$
\begin{aligned}
& \int_{\Omega}\left|\sigma\left(x, u_{k}, D u_{k}\right)\right|^{p^{\prime}(x)} d x \\
& \quad \leq C \int_{\Omega}|a(x)|^{p^{\prime}(x)}+\left|u_{k}\right|^{q(x) p^{\prime}(x)}+\left|D u_{k}\right|^{(p(x)-1) p^{\prime}(x)} d x<C .
\end{aligned}
$$

Thus $\left\|\left|\sigma\left(x, u_{k}, D u_{k}\right)\right|\right\|_{p^{\prime}(x), \Omega^{\prime}}$ is bounded by Lemma 2.2. $\int_{\Omega^{\prime}}|D u|^{p(x)} d x$ is arbitrarily small if the measure of $\Omega^{\prime}$ is chosen small enough, and so is $\||D u|\|_{p(x), \Omega^{\prime}}$ by Lemma 2.2. The 
equiintegrability of the sequence $\left\{I_{k, 3}\right\}$ can be obtained also by the boundedness of $\left\{u_{k}\right\}$. We can get the equiintegrability of $I_{k, 1}^{-}$by observing that

$$
\sigma\left(x, u_{k}, D u_{k}\right): D u_{k} \geq-b(x)+c_{2}\left|D u_{k}\right|^{p(x)} \geq-b(x)
$$

and moreover

$$
\int_{\Omega^{\prime}} I_{k, 1}^{-} d x \leq \int_{\Omega^{\prime}}|b(x)| d x
$$

By equation (3.2) we can infer from Lemma 3.5 that equation (5.1) holds.

Next we will prove that $X \leq 0$. Define $\operatorname{dist}\left(u, V_{k}\right)=\inf _{v \in V_{k}}\|u-v\| \|$ and fix $\varepsilon>0$. Then, there exists $k_{0} \in \mathbb{N}$ such that $\operatorname{dist}\left(u, V_{k}\right)<\varepsilon$ for any $k>k_{0}$, or equivalently,

$$
\begin{aligned}
\operatorname{dist}\left(u_{k}-u, V_{k}\right) & =\inf _{v \in V_{k}}\left\|u_{k}-u-v\right\| \| \\
& =\inf _{w \in V_{k}}\|u-w\|=\operatorname{dist}\left(u, V_{k}\right)<\varepsilon,
\end{aligned}
$$

for any $k>k_{0}$. Consequently, for $v_{k} \in V_{k}$, by Lemma 4.4 we may estimate $X$ as follows:

$$
\begin{aligned}
X & =\liminf _{k \rightarrow \infty} \int_{\Omega} \sigma\left(x, u_{k}, D u_{k}\right):\left(D u_{k}-D u\right) d x \\
& =\liminf _{k \rightarrow \infty}\left(\int_{\Omega} \sigma\left(x, u_{k}, D u_{k}\right): D\left(u_{k}-u-v_{k}\right) d x+\int_{\Omega} \sigma\left(x, u_{k}, D u_{k}\right): D v_{k} d x\right) \\
& \leq \liminf _{k \rightarrow \infty} 2\left\|\left|\sigma\left(x, u_{k}, D u_{k}\right)\right|\right\|_{p^{\prime}(x)} \cdot\left\|\left|D\left(u_{k}-u-v_{k}\right)\right|\right\|_{p(x)}+\left\langle f, v_{k}\right\rangle .
\end{aligned}
$$

The term $\left\|\left|\sigma\left(x, u_{k}, D u_{k}\right)\right|\right\|_{p^{\prime}(x)}$ is bounded by the growth condition (H2). On the other hand, by choosing $v_{k} \in V_{k}$ in such a way that $\left\|u_{k}-u-v_{k}\right\| \mid<2 \varepsilon$ for any $k>k_{0}$, the term $\left\|D\left(u_{k}-u-v_{k}\right)\right\|_{p(x)}$ is bounded by $2 \varepsilon$. Moreover, we have

$$
\left|\left\langle f, v_{k}\right\rangle\right| \leq\left|\left\langle f, v_{k}-\left(u_{k}-u\right)\right\rangle\right|+\left|\left\langle f, u_{k}-u\right\rangle\right| \leq 2 \varepsilon\|f\|_{-1, p^{\prime}(x)}+o(1) .
$$

Since $\varepsilon>0$ is arbitrary, this proves $X \leq 0$. We conclude from equation (5.2) that equation (5.1) holds.

At last we get the equiintegrability of $\sigma\left(x, u_{k}, D u_{k}\right)$ from

$$
\left|\int_{\Omega^{\prime}} \sigma\left(x, u_{k}, D u_{k}\right) d x\right| \leq \int_{\Omega^{\prime}}\left|\sigma\left(x, u_{k}, D u_{k}\right)\right| \cdot 1 d x \leq 2\left\|\left|\sigma\left(x, u_{k}, D u_{k}\right)\right|\right\|_{p^{\prime}(x), \Omega^{\prime}} \cdot\|1\|_{p(x), \Omega^{\prime}}
$$

where $\left\|\left|\sigma\left(x, u_{k}, D u_{k}\right)\right|\right\|_{p^{\prime}(x), \Omega^{\prime}}$ is bounded by equation (5.3) and the term $\|1\|_{p(x), \Omega^{\prime}}$ is arbitrarily small if the measure of $\Omega^{\prime}$ is chosen small enough.

Lemma 5.2 If equation (5.1) holds, $v_{x}$ is a probability measure for almost every $x \in \Omega$, and $\left\langle v_{x}, I\right\rangle=D u(x)$, we find that for almost every $x \in \Omega$

$$
(\sigma(x, u, \lambda)-\sigma(x, u, D u)):(\lambda-D u)=0 \quad \text { on } \operatorname{supp} v_{x}
$$


Proof Notice that

$$
\begin{aligned}
& \int_{\Omega} \int_{\mathbb{M}^{m \times n}} \sigma(x, u, D u):(\lambda-D u) d v_{x}(\lambda) d x \\
& \quad=\int_{\Omega} \int_{\mathbb{M}^{m \times n}} \sigma(x, u, D u): \lambda d v_{x}(\lambda) d x-\int_{\Omega} \int_{\mathbb{M}^{m \times n}} \sigma(x, u, D u): D u d v_{x}(\lambda) d x \\
& \quad=\int_{\Omega} \sigma(x, u, D u): \int_{\mathbb{M}^{m \times n}} \lambda d v_{x}(\lambda) d x-\int_{\Omega} \sigma(x, u, D u): D u \int_{\mathbb{M}^{m \times n}} 1 d v_{x}(\lambda) d x \\
& \quad=0 .
\end{aligned}
$$

We infer from equation (5.1) that

$$
\int_{\Omega} \int_{\mathbb{M}^{m \times n}}(\sigma(x, u, \lambda)-\sigma(x, u, D u)):(\lambda-D u) d \nu_{x}(\lambda) d x \leq 0
$$

By the monotonicity of $\sigma$, the integrand in the above inequality is nonnegative. It follows that for almost every $x \in \Omega$

$$
(\sigma(x, u, \lambda)-\sigma(x, u, D u)):(\lambda-D u)=0 \quad \text { on } \operatorname{supp} v_{x}
$$

Lemma 5.3 If $u_{k} \rightarrow u$ in $W_{0}^{1, p(x)}\left(\Omega, \mathbb{R}^{m}\right)$ and $\sigma$ satisfies $(\mathrm{H} 1)-(\mathrm{H} 3)$, then for any $v \in$ $W_{0}^{1, p(x)}\left(\Omega, \mathbb{R}^{m}\right)$, we have

$$
\int_{\Omega}\left(\sigma\left(x, u_{k}, D u_{k}\right): D v-\sigma(x, u, D u): D v\right) d x \rightarrow 0 \text { as } k \rightarrow \infty .
$$

Proof By Theorem 3.7, the sequence $\left\{\left(u_{k}, D u_{k}\right)\right\}$ generates the Young measure $\delta_{u(x)} \otimes v_{x}$ and for almost every $x \in \Omega, v_{x}$ is a probability measure such that $\left\langle v_{x}, I\right\rangle=D u(x)$. The proof will be divided into three cases. In the following, cases (i)-(iii) correspond to the three cases of (H3).

Case (i): We claim that in this case for almost every $x \in \Omega$ and for every $\mu \in \mathbb{M}^{m \times n}$ the following identity holds on $\operatorname{supp} v_{x}$ :

$$
\sigma(x, u, \lambda): \mu=\sigma(x, u, D u): \mu+(\nabla \sigma(x, u, D u) \mu):(D u-\lambda)
$$

where $\nabla$ is the derivative with respect to the third variable of $\sigma$. Indeed, by the monotonicity of $\sigma$ we have for each $t \in \mathbb{R}$

$$
(\sigma(x, u, \lambda)-\sigma(x, u, D u+t \mu)):(\lambda-D u-t \mu) \geq 0,
$$

and, by Lemma 5.2,

$$
\begin{aligned}
& \sigma(x, u, \lambda):(\lambda-D u)-\sigma(x, u, \lambda): t \mu-\sigma(x, u, D u+t \mu):(\lambda-D u-t \mu) \\
& \quad=\sigma(x, u, D u):(\lambda-D u)-\sigma(x, u, \lambda): t \mu-\sigma(x, u, D u+t \mu):(\lambda-D u-t \mu) .
\end{aligned}
$$

Therefore

$$
-\sigma(x, u, \lambda): t \mu \geq-\sigma(x, u, D u):(\lambda-D u)+\sigma(x, u, D u+t \mu):(\lambda-D u-t \mu)
$$


and

$$
\sigma(x, u, D u+t \mu)=\sigma(x, u, D u)+\nabla \sigma(x, u, D u) t \mu+o(t) .
$$

Thus

$$
\begin{aligned}
& \sigma(x, u, D u+t \mu):(\lambda-D u-t \mu) \\
&= \sigma(x, u, D u+t \mu):(\lambda-D u)-\sigma(x, u, D u+t \mu): t \mu \\
&= \sigma(x, u, D u):(\lambda-D u)+\nabla \sigma(x, u, D u) t \mu:(\lambda-D u) \\
& \quad-\sigma(x, u, D u): t \mu+\nabla \sigma(x, u, D u) t \mu: t \mu+o(t) \\
&= \sigma(x, u, D u):(\lambda-D u)+t(\nabla \sigma(x, u, D u) \mu:(\lambda-D u)-\sigma(x, u, D u): \mu)+o(t) .
\end{aligned}
$$

Then we get

$$
-\sigma(x, u, \lambda): t \mu \geq t((\nabla \sigma(x, u, D u) \mu)(\lambda-D u)-\sigma(x, u, D u): \mu)+o(t) .
$$

Equation (5.4) follows from this inequality since the sign of $t$ is arbitrary. Take $\mu=E_{i j}$, where $E_{i j}$ is the matrix whose entry in the $i$ th row and $j$ th column is 1 and others are 0 . Then by equation (5.4),

$$
\sigma(x, u, \lambda)_{i j}=\sigma(x, u, D u)_{i j}+(\nabla \sigma(x, u, D u) \mu)_{i j}:(D u-\lambda),
$$

further we can get

$$
\begin{aligned}
\int_{\operatorname{supp} v_{x}} \sigma(x, u, \lambda)_{i j} d v_{x}(\lambda)= & \int_{\operatorname{supp} v_{x}} \sigma(x, u, D u)_{i j} d v_{x}(\lambda) \\
& +(\nabla \sigma(x, u, D u) \mu)_{i j}: \int_{\operatorname{supp} v_{x}}(D u-\lambda) d v_{x}(\lambda) .
\end{aligned}
$$

Notice that $\int_{\operatorname{supp} v_{x}}(D u-\lambda) d v_{x}(\lambda)=0$, thus

$$
\int_{\operatorname{supp} v_{x}} \sigma(x, u, \lambda) d v_{x}(\lambda)=\int_{\operatorname{supp} v_{x}} \sigma(x, u, D u) d v_{x}(\lambda)=\sigma(x, u, D u) .
$$

Since equation (5.3) and Lemma 5.1 imply that the sequence $\left\{\sigma\left(x, u_{k}, D u_{k}\right)\right\}$ is bounded and equiintegrable, by the Dunford-Pettis criterion and Lemma 3.2 its weak $L^{1}$-limit $\bar{\sigma}$ is given by

$$
\bar{\sigma}=\int_{\operatorname{supp} v_{x}} \sigma(x, u, \lambda) d v_{x}(\lambda)=\sigma(x, u, D u) .
$$

By Lemma 2.3 , the sequence $\left\{\sigma\left(x, u_{k}, D u_{k}\right)\right\}$ converges weakly in $L^{p^{\prime}(x)}\left(\Omega, \mathbb{M}^{m \times n}\right)$. Hence its weak $L^{p^{\prime}(x)}$-limit is also $\sigma(x, u, D u)$. Then we conclude that

$$
\int_{\Omega}\left(\sigma\left(x, u_{k}, D u_{k}\right): D v-\sigma(x, u, D u): D v\right) d x \rightarrow 0 \quad \text { as } k \rightarrow \infty
$$


Case (ii): We start by showing that for almost every $x \in \Omega$,

$$
\operatorname{supp} v_{x} \subset K_{x}=\left\{\lambda \in \mathbb{M}^{m \times n}: W(x, u, \lambda)=W(x, u, D u)+\sigma(x, u, D u):(\lambda-D u)\right\}
$$

If $\lambda \in \operatorname{supp} v_{x}$, by Lemma 5.2

$$
(1-t)(\sigma(x, u, \lambda)-\sigma(x, u, D u)):(\lambda-D u)=0 \quad \text { for all } t \in[0,1] .
$$

On the other hand, by monotonicity, for $t \in[0,1]$ we have

$$
(1-t)(\sigma(x, u, D u+t(\lambda-D u))-\sigma(x, u, \lambda)):(D u-\lambda) \geq 0 .
$$

Subtracting equation (5.5) from equation (5.6), we get

$$
(1-t)(\sigma(x, u, D u+t(\lambda-D u))-\sigma(x, u, D u)):(D u-\lambda) \geq 0
$$

for any $t \in[0,1]$. By monotonicity,

$$
(\sigma(x, u, D u+t(\lambda-D u))-\sigma(x, u, D u)): t(\lambda-D u) \geq 0 .
$$

Since $t \in[0,1]$, we have

$$
(\sigma(x, u, D u+t(\lambda-D u))-\sigma(x, u, D u)):(1-t)(\lambda-D u) \geq 0 .
$$

Then we have

$$
(\sigma(x, u, D u+t(\lambda-D u))-\sigma(x, u, D u)):(\lambda-D u)=0
$$

for any $t \in[0,1]$, whenever $\lambda \in \operatorname{supp} v_{x}$. Now, it follows from equation (5.8) that

$$
\begin{aligned}
W(x, u, \lambda) & =W(x, u, D u)+\int_{0}^{1} \sigma(x, u, D u+t(\lambda-D u)):(\lambda-D u) d t \\
& =W(x, u, D u)+\sigma(x, u, D u):(\lambda-D u) .
\end{aligned}
$$

Thus we can conclude that $\lambda \in K_{x}$, i.e. $\operatorname{supp} v_{x} \subset K_{x}$.

By the convexity of $W$ we have $W(x, u, \xi) \geq W(x, u, D u)+\sigma(x, u, D u):(\xi-D u)$ for any $\xi \in \mathbb{M}^{m \times n}$. For every $\lambda \in K_{x}$, we set $P(\lambda)=W(x, u, \lambda), Q(\lambda)=W(x, u, D u)+\sigma(x, u, D u):(\lambda-$ $D u)$. Since the mapping $\lambda \rightarrow W(x, u, \lambda)$ is continuously differentiable, for every $\varphi \in \mathbb{M}^{m \times n}$, $t \in \mathbb{R}$

$$
\begin{array}{ll}
\frac{P(\lambda+t \varphi)-P(\lambda)}{t} \geq \frac{Q(\lambda+t \varphi)-Q(\lambda)}{t} & (t>0), \\
\frac{P(\lambda+t \varphi)-P(\lambda)}{t} \leq \frac{Q(\lambda+t \varphi)-Q(\lambda)}{t} & (t<0) .
\end{array}
$$

Hence $D P=D Q$ and we obtain

$$
\sigma(x, u, \lambda)=\sigma(x, u, D u) \quad \text { for any } \lambda \in K_{x} \supset \operatorname{supp} v_{x} .
$$


Consequently

$$
\begin{aligned}
\bar{\sigma}(x) & :=\int_{\mathbb{M}^{m} \times n} \sigma(x, u, \lambda) d v_{x}(\lambda) \\
& =\int_{\operatorname{supp} v_{x}} \sigma(x, u, \lambda) d v_{x}(\lambda)=\sigma(x, u, D u) .
\end{aligned}
$$

Now consider the Carathéodory function

$$
g(x, \zeta, \lambda)=|\sigma(x, \zeta, \lambda)-\bar{\sigma}(x)|, \quad \zeta \in \mathbb{R}^{m}, \lambda \in \mathbb{M}^{m \times n}
$$

The sequence $g_{k}(x)=g\left(x, u_{k}(x), D u_{k}(x)\right)$ is equiintegrable, so

$$
g_{k} \rightarrow \bar{g} \quad \text { weakly in } L^{1}(\Omega)
$$

and the weak limit $\bar{g}$ is

$$
\begin{aligned}
\bar{g}(x) & =\int_{\mathbb{R}^{m} \times \mathbb{M}^{m \times n}}|\sigma(x, \zeta, \lambda)-\bar{\sigma}(x)| d \delta_{u(x)}(\zeta) \otimes d v_{x}(\lambda) \\
& =\int_{\operatorname{supp} v_{x}}|\sigma(x, u(x), \lambda)-\bar{\sigma}(x)| d v_{x}(\lambda) \\
& =\int_{\operatorname{supp} v_{x}}|\sigma(x, u(x), \lambda)-\sigma(x, u(x), D u(x))| d v_{x}(\lambda)=0
\end{aligned}
$$

by equations (5.9) and (5.10). Then

$$
\int_{\Omega}\left|\sigma\left(x, u_{k}(x), D u_{k}(x)\right)-\sigma(x, u(x), D u(x))\right| d x \rightarrow 0 .
$$

Therefore by Vitali's theorem

$$
\int_{\Omega}\left(\sigma\left(x, u_{k}, D u_{k}\right): D v-\sigma(x, u, D u): D v\right) d x \rightarrow 0 \quad \text { as } k \rightarrow \infty
$$

Case (iii): By strict monotonicity, it follows from Lemma 5.2 that supp $v_{x}=\{D u(x)\}$, thus $v_{x}=\delta_{D u(x)}$ for almost every $x \in \Omega$. By Lemma 3.3 and $D u_{k} \rightarrow D u$ in the measure and by equation (3.2) $u_{k} \rightarrow u$ in the measure. After extracting a suitable subsequence if necessary, we can infer that $D u_{k} \rightarrow D u$ for almost every $x \in \Omega$ and $u_{k} \rightarrow u$ for almost every $x \in \Omega$. Then $\sigma\left(x, u_{k}, D u_{k}\right) \rightarrow \sigma(x, u, D u)$ for almost every $x \in \Omega$, moreover we have $\sigma\left(x, u_{k}, D u_{k}\right) \rightarrow \sigma(x, u, D u)$ in the measure. By the equiintegrability of $\sigma\left(x, u_{k}, D u_{k}\right): D v$, already discussed above, the Vitali theorem implies

$$
\int_{\Omega}\left(\sigma\left(x, u_{k}, D u_{k}\right): D v-\sigma(x, u, D u): D v\right) d x \rightarrow 0 \quad \text { as } k \rightarrow \infty
$$

The proof of Lemma 5.3 is completed.

Proof of Theorem 1.1 It is sufficient to prove that for any $v \in W_{0}^{1, p(x)}\left(\Omega, \mathbb{R}^{m}\right)$ there is $u \in$ $W_{0}^{1, p(x)}\left(\Omega, \mathbb{R}^{m}\right)$ such that $\langle J(u), v\rangle=0$. 
From the coercivity assumption in (H2) and Lemma 4.3, it follows that there exists $R>0$ such that $\langle(u), u\rangle>1$ whenever $\|u\|>R$. Thus, for the sequence of Galerkin approximations $u_{k} \in V_{k}$ constructed in Lemma 4.4, we have

$$
\left\|u_{k}\right\| \mid \leq R \quad \text { for all } k
$$

Then we may extract a subsequence (still denoted by $u_{k}$ ) such that

$$
u_{k} \rightarrow u \quad \text { in } W_{0}^{1, p(x)}\left(\Omega, \mathbb{R}^{m}\right)
$$

For any $v \in W_{0}^{1, p(x)}\left(\Omega, \mathbb{R}^{m}\right)$, since $\bigcup_{i \in \mathbb{N}} V_{i}$ is dense in $W_{0}^{1, p(x)}\left(\Omega, \mathbb{R}^{m}\right)$, there is a sequence $\left\{v_{k}\right\} \subset \bigcup_{i \in \mathbb{N}} V_{i}$ such that $v_{k} \rightarrow v$ in $W_{0}^{1, p(x)}\left(\Omega, \mathbb{R}^{m}\right)$ as $k \rightarrow \infty$. By Lemma 5.3 , we have

$$
\begin{aligned}
& \left\langle J\left(u_{k}\right), v_{k}\right\rangle-\langle J(u), v\rangle \\
& =\int_{\Omega}\left(\sigma\left(x, u_{k}, D u_{k}\right): D v_{k}-\sigma\left(x, u_{k}, D u_{k}\right): D v\right. \\
& \left.\quad+\sigma\left(x, u_{k}, D u_{k}\right): D v-\sigma(x, u, D u): D v\right) d x+\left\langle f, v_{k}-v\right\rangle \\
& \quad \rightarrow 0
\end{aligned}
$$

as $k \rightarrow \infty$. Lemma 4.4 implies that $\langle J(u), v\rangle=0$ for all $v \in W_{0}^{1, p(x)}\left(\Omega, \mathbb{R}^{m}\right)$.

\section{Competing interests}

The authors declare that they have no competing interests.

\section{Authors' contributions}

The authors declare that the study was realized in collaboration with the same responsibility. All authors read and approved the final manuscript.

\section{Acknowledgements}

This work was supported by NSFC-11371110.

Received: 17 May 2013 Accepted: 17 December 2013 Published: 14 Jan 2014

\section{References}

1. Kováčik, O, Rákosník, J: On spaces $L^{p(x)}$ and $W^{m, p(x)}$. Czechoslov. Math. J. 41(116), 592-618 (1991)

2. Edmunds, D, Lang, J, Nekvinda, A: On $L^{p(x)}$ norms. Proc. R. Soc. Lond., Ser. A, Math. Phys. Eng. Sci. 455, $219-225$ (1999)

3. Edmunds, D, Rákosník, J: Sobolev embedding with variable exponent. Stud. Math. 143, 267-293 (2000)

4. Fan, X, Shen, J, Zhao, D: Sobolev embedding theorems for spaces $W^{m}$ p (x) . J. Math. Anal. Appl. 262, 749-760 (2001)

5. Fan, $X$, Zhao, D: On the spaces $L^{p(x)}$ and $W^{m, p(x)}$. J. Math. Anal. Appl. 263, 424-446 (2001)

6. Diening, L, Harjulehto, P, Hästö, P, Růžička, M: Lebesgue and Sobolev Spaces with Variable Exponents. Lecture Notes in Mathematics, vol. 2017. Springer, Berlin (2011)

7. Chabrowski, J, Fu, Y: Existent of solutions for $p(x)$-Laplacian problems on a bounded domain. J. Math. Anal. Appl. 306, 604-618 (2005). (Corrigendum: J. Math. Anal. Appl. 323, 1483 (2006))

8. Fan, X, Zhang, Q, Zhao, D: Eigenvalues of $p(x)$-Laplacian Dirichlet problem. J. Math. Anal. Appl. 302, $306-317$ (2005)

9. Galewski, M: New variational method for $p(x)$-Laplacian equation. Bull. Aust. Math. Soc. 72, 53-65 (2005)

10. Acerbi, E, Mingione, G: Regularity results for stationary electro-rheological fluids. Arch. Ration. Mech. Anal. 164, 213-259 (2002)

11. Acerbi, E, Mingione, G, Seregin, GA: Regularity results for parabolic systems related to a class of non-Newtonian fluids. Ann. Inst. Henri Poincaré, Anal. Non Linéaire 21, 25-60 (2004)

12. Mihăilescu, B, Radulescu, V: A multiplicity result for a nonlinear degenerate problem arising in the theory of electrorheological fluids. Proc. R. Soc. Lond., Ser. A, Math. Phys. Eng. Sci. 462, 2625-2641 (2006)

13. Chen, YM, Levine, S, Rao, M: Variable exponent linear growth functionals in image restoration. SIAM J. Appl. Math. 66, 1383-1406 (2006)

14. Zhikov, V: Averaging of functionals in the calculus of variations and elasticity. Math. USSR, Izv. 29, 33-66 (1987)

15. Antontsev, SN, Shmarev, SI: A model porous medium equation with variable exponent of nonlinearity: existence, uniqueness and localization properties of solutions. Nonlinear Anal. 60, 515-545 (2005) 
16. Hungerbühler, N: Quasilinear elliptic systems in divergence form with weak monotonicity. N.Y. J. Math. 5, 83-90 (1999)

17. Višik, Ml: Quasilinear strongly elliptic systems of differential equations of divergence form. Tr. Mosk. Mat. Obŝ. 12, 125-184 (1963)

18. Minty, GJ: Monotone (nonlinear) operators in Hilbert space. Duke Math. J. 29, 341-346 (1962)

19. Brézis, H: Operateurs Maximaux Monotones et Semigroups de Contractions dans les Spaces de Hilbert. North-Holland, Amsterdam (1973)

20. Lions, JL: Quelques Méthodes de Résolution des Problèmes aux Limites non Linéaires. Gauthier-Villars, Paris (1969)

21. Dolzmann, G, Hungerbühler, N, Müller, S: Nonlinear elliptic systems with measure-valued right hand side. Math. Z. 226, 545-574 (1997)

22. Evans, LC: Weak Convergence Methods for Nonlinear Partial Differential Equations. Am. Math. Soc., New York (1990)

23. Ball, JM: A version of the fundamental theorem for Young measures. In: PDEs and Continuum Models of Phase Transitions (Nice, 1988). Lecture Notes in Phys., vol. 344, pp. 207-215. Springer, Berlin (1989)

24. Hungerbühler, N: A refinement of Ball's theorem on Young measures. N.Y. J. Math. 3, 48-53 (1997)

25. Nirenberg, L: Topics in Nonlinear Functional Analysis. Lecture Notes. Courant Institute, New York (1974)

10.1186/1029-242X-2014-23

Cite this article as: Fu and Yang: Existence of solutions for quasilinear elliptic systems in divergence form with

variable growth. Journal of Inequalities and Applications 2014, 2014:23

\section{Submit your manuscript to a SpringerOpen ${ }^{\odot}$ journal and benefit from:}

- Convenient online submission

- Rigorous peer review

Immediate publication on acceptance

- Open access: articles freely available online

- High visibility within the field

- Retaining the copyright to your article 